\title{
Laparoscope- Lots of Hope And Scope
}

\author{
Dr.Potluri lakshmi tejeswini \\ Under The Guidance Of Prof.N.Palaniappan And Dr.Priyanka Mehta
}

\section{Introduction}

Torsion of ovary is the total or partial rotation of the adnexa around its vascular axis or pedicle. Complete torsion causes venous and lymphatic blockade leading to stasis and venous congestion, haemorrhage and necrosis.

The cyst becomes tense and may rupture.

Incidence is 5 per 10,000 pregnancies.

Torsion is predominantly seen in the reproductive age group and in women after ART.

\section{Case Report}

25 year old primi at $27 \mathrm{w}+2 \mathrm{~d}$, presented with acute pain abdomen since one day.

OI conception.

Her first \& second trimesters were uneventful.

USG showed right ovary appears edematous measuring $6.2 \times 5.2 \mathrm{~cm}$ with minimal vascularity noted, there is no ultrasound evidence of torsion.

Examination revealed involuntary guarding and rigidity of the right side of her abdomen.

No history of vaginal bleeding.

O/E: Patient was afebrile

\section{Investigations}

Bp-120/80 mmhg

Pr- $88 / \mathrm{min}$

$\mathrm{Rr}-18 / \mathrm{min}$

P/A- soft

Gaurding +

Rigidity +

Right iliac fossa-Tenderness +

Cephalic,FH +

Acute appendicitis was suspected and a prompt review by the surgical team was undertaken.

USG growth scan done showed single live intrauterine gestation corresponding to 27 weeks +2 days, placenta anterior, estimated fetal weight $1.07 \mathrm{~kg}$ with right ovary appears edematous measuring $6.2 \times 5.2 \mathrm{~cm}$ with minimal vascularity noted, there is no ultrasound evidence of torsion.

AFP-261.90

CA $125-9.50$

Urine Culture- No growth

\section{Differential Diagnosis}

Appendicitis

Degenerating fibroid

Ovarian torsion.

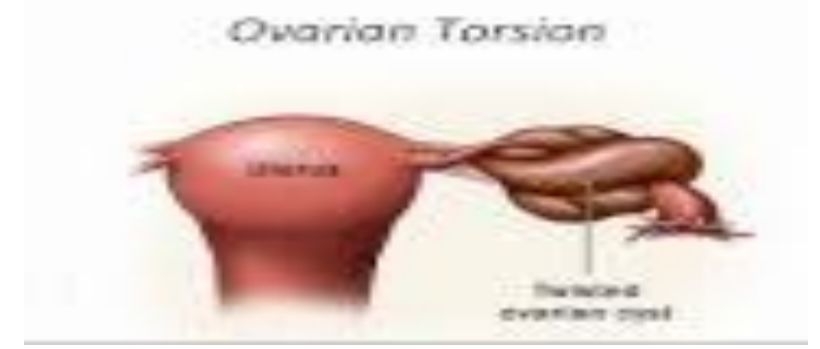




\section{Treatment}

Emergency Laproscopic Right Oophrectomy With Partial Salphingectomy Done.

Intra op findings: Right ovary enlarged 6 x $6 \mathrm{~cm}$ (torsion - 4 times and necrosed).

Left side tubes and ovaries normal.

$\mathrm{FH}+$ checked by Doppler post operatively.

Histopathology: Benign serous cyst with changes secondary to torsion.

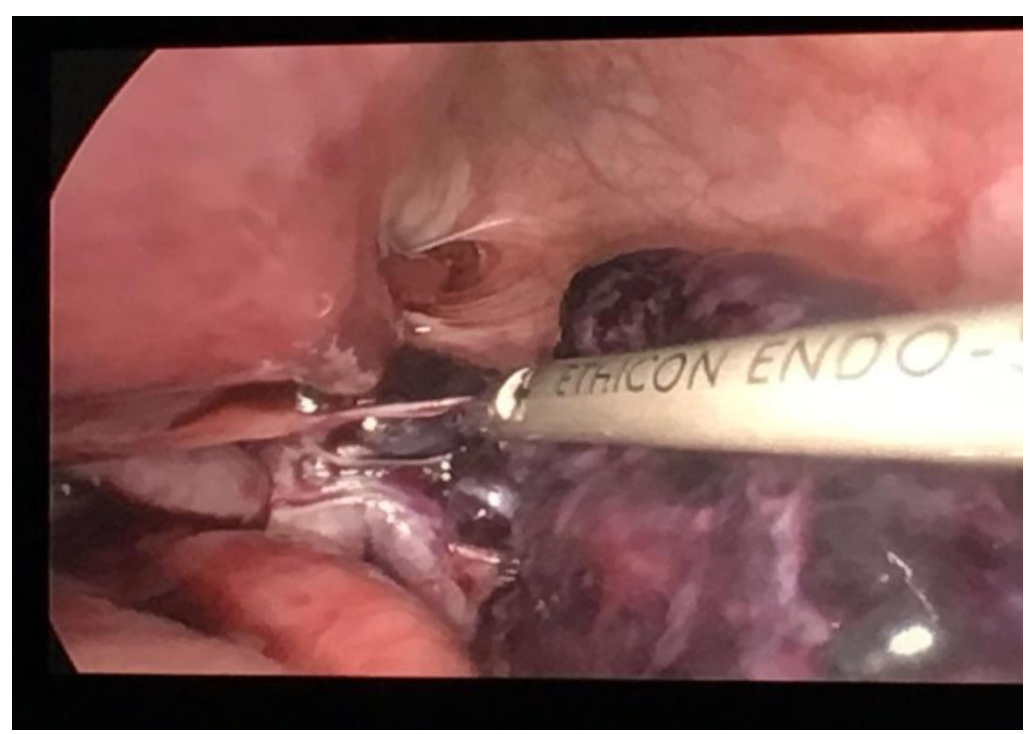

V. Result

Patient was followed up. She delivered a healthy girl baby at 39 weeks by svd with epi

\section{Discussion}

Benign dermoid cysts/teratomas are the most frequent ovarian tumors, with an incidence ranging from 5\% to $25 \%$ of all ovarian neoplasms (1). They are of germ cell origin and composed of multiple types of tissue. Torsion of the cystic contents and ovary may occur in them, thus leading to vascular infarction and necrosis. Torsion of the pedicle has been reported to be the most frequent complication, occurring in $16.1 \%$ of cases (1). Traditional risk factors for ovarian torsion are increased ovarian size, ovarian tumors, ovarian hyperstimulation, and pregnancy $(2,3,4)$. Torsion of the ovary in the third trimester is rare as the compressive effect of the gravid uterus restricts the mobility of the ovarian pedicle. However this case clearly demonstrates that it can occur and needs to be considered as a differential diagnosis when patients present with an acute abdomen. Although conservative treatment has been proposed during pregnancy, surgical intervention is the treatment of choice once ovarian torsion is highly suspected [5]. Ultrasound scan examinations in early pregnancy should also address the cervix and the adnexa leading to early diagnosis and management of ovarian masses, thus avoiding later emergency situations and the possibility of preterm deliveries. Ultrasonographic imaging during pregnancy is safe and useful in identifying the etiology of acute abdominal pain in the pregnant patient(6).

\section{Society of American Gastrointestinal and Endoscopic Surgeons-SAGES}

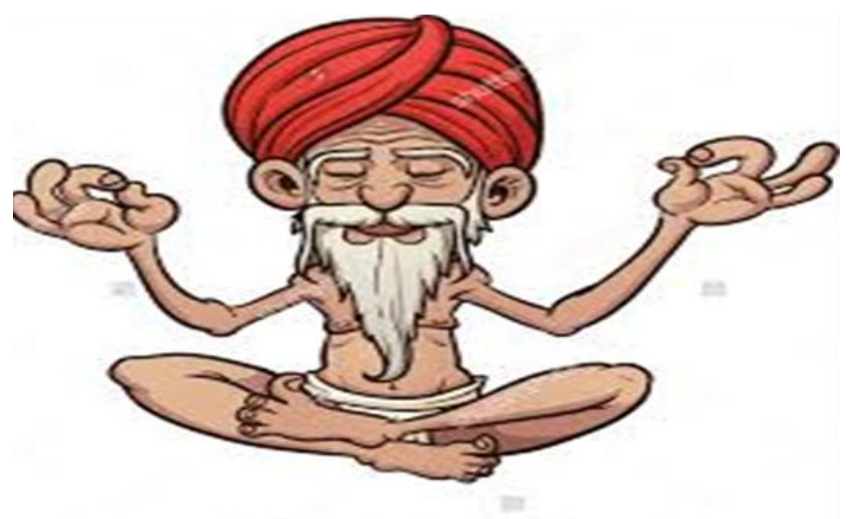


Guideline 8: Laparoscopic treatment of acute abdominal disease has the same indications in pregnant and non-pregnant patients

Guideline 9: Laparoscopy can be safely performed during any trimester of pregnancy

Guideline 10: Gravid patients should be placed in the left lateral decubitus position to minimize compression of the vena cava

Guideline 11: Initial abdominal access can be safely accomplished with an open (Hasson) technique, Veress needle or optical trocar, if the location is adjusted according to fundal height and previous incisions

Guideline 12: CO2 insufflation of $10-15 \mathrm{mmHg}$ can be safely used for laparoscopy in the pregnant patient

\section{Conclusion}

Diagnosis can usually be made on the basis of signs and symptoms along with USG findings. Treatment options are limited to surgery.

\section{References}

[1]. E. Shalev, M. Bustan, S. Romano, Y. Goldberg, and I. Ben-Shlomo, "Laparoscopic resection of ovarian benign cystic teratomas: experience with 84 cases," Human Reproduction, vol. 13, no. 7, pp. 1810-1812, 1998.

[2]. P.-H. Wang, H.-T. Chao, C.-C. Yuan, W.-L. Lee, K.-C. Chao, and H.-T. Ng, "Ovarian tumors complicating pregnancy. Emergency and elective surgery," Journal of Reproductive Medicine for the Obstetrician and Gynecologist, vol. 44, no. 3, pp. 279-287, 1999.

[3]. P.-H. Wang, W.-H. Chang, M.-H. Cheng, and H.-C. Horng, "Management of adnexal masses during pregnancy," Journal of Obstetrics and Gynaecology Research, vol. 35, no. 3, pp. 597-598, 2009.

[4]. H.-C. Tsai, T.-N. Kuo, M.-T. Chung, M. Y. S. Lin, C.-Y. Kang, and Y.-C. Tsai, "Acute abdomen in early pregnancy due to ovarian torsion following successful in vitro fertilization treatment," Taiwanese Journal of Obstetrics and Gynecology, vol. 54, no. 4, pp. 438-441, 2015.

[5]. S. Krishnan, H. Kaur, J. Bali, and K. Rao, "Ovarian torsion in infertility management - missing the diagnosis means losing the ovary: a high price to pay," Journal of Human Reproductive Sciences, vol. 4, no. 1, pp. 39-42, 2011.

[6]. Guidelines of Society of American Gastrointestinal and Endoscopic Surgeons (SAGES) on Sep 2007 